\title{
Experimental Studies on the Lorentz Symmetry in Post-Newtonian Gravity with Pulsars ${ }^{+}$
}

\author{
Lijing Shao \\ Max Planck Institute for Gravitational Physics (Albert Einstein Institute), Am Mühlenberg 1, \\ D-14476 Potsdam-Golm, Germany; lijing.shao@aei.mpg.de \\ † Varying Constants and Fundamental Cosmology_VARCOSMOFUN'16. \\ Academic Editors: Mariusz P. Dąbrowski, Manuel Krämer and Vincenzo Salzano \\ Received: 30 October 2016; Accepted: 23 November 2016; Published: 1 December 2016
}

\begin{abstract}
Local Lorentz invariance (LLI) is one of the most important fundamental symmetries in modern physics. While the possibility of LLI violation (LLIv) was studied extensively in flat spacetime, its counterpart in gravitational interaction also deserves significant examination from experiments. In this contribution, I review several recent studies of LLI in post-Newtonian gravity, using powerful tools of pulsar timing. It shows that precision pulsar timing experiments hold a unique position to probe LLIv in post-Newtonian gravity.
\end{abstract}

Keywords: local Lorentz invariance; pulsar astronomy; tests of gravity

\section{Introduction}

In 1905, one of Einstein's annus mirabilis papers_-"On the electrodynamics of moving bodies" (in German, "Zur Elektrodynamik bewegter Körper")—permanently established the Lorentzian nature of a flat spacetime in special relativity, and it was later extended by Einstein himself to general relativity (GR) locally. According to GR, for every point on our 4-dimensional spacetime manifold, its tangent space possesses the symmetry of a Lorentz group. As a foundational principle of today's theoretical physics, the local Lorentz invariance (LLI) deserves empirical examination with exquisite precision [1-5].

When performing phenomenological studies to test the Lorentz symmetry, if gravitation is involved, one shall carefully distinguish the concept of Lorentz symmetry in flat and curved spacetimes $[1,2,6]$. The LLI violation (LLIv) in gravitational interaction modifies the local Lorentzian transformation property of gravitation, and leads to various anomalous phenomena in gravitational experiments [2,3,7-11], including lunar laser ranging [12,13], atom interferometry [14], pulsar timing [15-19], cosmic rays [20,21], very long baseline interferometry [22], and short-range experiments in laboratory [23-26]. In this short contribution, I will focus on the recent limits on LLIv obtained from precision pulsar timing experiments $[7,15-19,27,28]$ in the theoretical frameworks of parametrized post-Newtonian (PPN) gravity [2,29] and the pure gravity sector of the standard-model extension (SME) [1,6,7]. Interested readers are encouraged to elaborate reviews for more details $[2-5,30]$.

\section{Parametrized Post-Newtonian Gravity of Will and Nordtvedt}

The PPN formalism was developed to conduct precision tests of post-Newtonian gravity in the solar system. It incorporates various ways, in terms of ten PPN parameters in different components of the metric $g^{\mu v}$, to challenge fundamental properties in GR, such as the amount of nonlinearity in gravity, the LLI, and the conservation laws of energy and momentum [2]. In the PPN 
formalism, the semi-conservative Lagrangian for the gravitational interaction of a binary pulsar with masses $m_{p}$ (pulsar) and $m_{\mathcal{c}}$ (companion) can be written as [2,15,16,29],

$$
L=L_{\beta, \gamma}+L_{\alpha_{1}}+L_{\alpha_{2}}
$$

where $L_{\beta, \gamma}$ is the post-Newtonian terms from GR and its minimal extensions characterized by the Eddington-Robertson-Schiff parameters, $\beta$ and $\gamma$,

$$
\begin{aligned}
L_{\beta, \gamma}= & -m_{p} \sqrt{1-\left(\mathbf{v}_{p}^{0}\right)^{2}}-m_{c} \sqrt{1-\left(\mathbf{v}_{c}^{0}\right)^{2}}+\frac{G m_{p} m_{c}}{r} \\
& +\frac{G m_{p} m_{c}}{r}\left[\frac{\left(\mathbf{v}_{p}^{0}\right)^{2}+\left(\mathbf{v}_{c}^{0}\right)^{2}}{2}-\frac{3 \mathbf{v}_{p}^{0} \cdot \mathbf{v}_{c}^{0}}{2}-\frac{\left(\mathbf{n} \cdot \mathbf{v}_{p}^{0}\right)\left(\mathbf{n} \cdot \mathbf{v}_{c}^{0}\right)}{2}+\gamma\left(\mathbf{v}_{p}^{0}-\mathbf{v}_{c}^{0}\right)^{2}-(2 \beta-1) \frac{G M}{2 r}\right],
\end{aligned}
$$

and the velocity-dependent, LLIv terms,

$$
\begin{aligned}
L_{\alpha_{1}} & =-\alpha_{1} \frac{G m_{p} m_{c}}{r} \frac{\mathbf{v}_{p}^{0} \cdot \mathbf{v}_{c}^{0}}{2}, \\
L_{\alpha_{2}} & =\alpha_{2} \frac{G m_{p} m_{c}}{r} \frac{\mathbf{v}_{p}^{0} \cdot \mathbf{v}_{c}^{0}-\left(\mathbf{n} \cdot \mathbf{v}_{p}^{0}\right)\left(\mathbf{n} \cdot \mathbf{v}_{c}^{0}\right)}{2},
\end{aligned}
$$

where $M \equiv m_{p}+m_{c}, r \equiv|\mathbf{r}|$ is the coordinate separation (in the PPN gauge) of two components, $\mathbf{n} \equiv \mathbf{r} / r, \mathbf{v}^{0}$ denotes the absolute velocity with respect to a preferred frame (see $[2,16]$ for more details). $\beta, \gamma, \alpha_{1}, \alpha_{2}$ are PPN parameters which take different numerical values in different alternative gravity theories; in GR, $\beta=\gamma=1$ and $\alpha_{1}=\alpha_{2}=0$ [2]. There is another PPN parameter, $\alpha_{3}$, that leads to LLIv in gravity; but, in addition, it violates energy-momentum conservation, thus is not included here [2].

It was found by Damour and Esposito-Farèse that, for a binary pulsar in a quasi-circular orbit, the existence of a nonzero $\alpha_{1}$ term (3) tends to polarize the binary's Laplace-Runge-Lenz vector according to the absolute velocity of the binary with respect to a cosmologically preferred frame [15]. Such an orbital polarization phenomenon can be studied with a population of small-eccentricity binary pulsars by assuming that, (i) they have been evolved for a long time to achieve the randomness in the orientation of the general-relativistically precessing Laplace-Runge-Lenz vector, (ii) and the longitude of ascending nodes is randomly distributed in $\left[0^{\circ}, 360^{\circ}\right)$ [30]. With these assumptions, one can obtain a probabilistic constraint on the value of $\alpha_{1}$ with a handful of binary pulsars [15]. Recently, the probabilistic method was extended into a robust one which drops these two assumptions [16]. The new method takes advantage of new observations which, by combining radio timing and optical spectroscopic data, give (i) the detailed information on the binary's 3-dimensional motion in space; (ii) a precision mass measurement which is independent of the underlying gravity theories; (iii) and a better measurement of the orbital Laplace-Runge-Lenz vector where its time variation can be inferred. A white-dwarf neutron-star binary, PSR J1738+0333, with an orbital period $\sim 8.5 \mathrm{~h}$ and an eccentricity $\sim 10^{-7}$ [31], turns out to be the best celestial laboratory at the current stage for this test, and gives the best limit on the PPN parameter $\left|\alpha_{1}\right| \lesssim 10^{-5}[2,16]$.

In the same publication, the effect of $\alpha_{2}$ on a binary pulsar was also worked out. It was found that, for a quasi-circular orbit, the effects of $\alpha_{1}$ and $\alpha_{2}$ decouple. A nonzero $\alpha_{2}$ term (4) tends to rotate the binary's orbital angular momentum around a direction according to the absolute velocity of the binary with respect to a preferred frame [16,32]. The change of a binary's projected semi-major axis from pulsar timing observation can be used to constrain the value of $\alpha_{2}$, but the constraint is weaker than the one obtained in the solar system by Nordtvedt [33]. Later, the idea of using a rotating massive body to constrain $\alpha_{2}$ [33] inspired the usage of solitary millisecond pulsars to test $\alpha_{2}$ [17]. It was found that the $N$-body version of Equation (4), from which the equations of motion for constituent particles of a neutron star can be derived, introduces a free precession of a rapidly 
spinning pulsar along an axis defined by the direction of the spinning axis and the absolute velocity of the pulsar $[17,33]$. The magnitude of the precession is inversely proportional to the spin period of the rotating body. Therefore well-timed millisecond pulsars are the best objects to study $\alpha_{2}$. In the case of a nonzero $\alpha_{2}$, the precession would introduce changes in the pulsar's pulse profile received on the Earth, which was not seen in the $\gtrsim 10$-year Effelsberg radio telescope's observation of PSRs B1937+21 and J1744-1134 [17]. Such a non-detection was translated into an extremely tight limit, $\left|\alpha_{2}\right| \lesssim 10^{-9}$ [17], which surpasses all previous limits [2]. As a side remark, the non-detection of the pulsar's pulse profile change was also extremely useful to constrain the Whitehead's term in the PPN formalism $[2,34]$.

\section{Standard-Model Extension of Kostelecký et al.}

In contrast to the metric-based approach in the PPN formalism, SME uses an action-based approach that introduces LLIv from a spontaneous symmetry breaking of vectorial and tensorial fields dynamically [6]. For the case of LLIv with energy-momentum conservation, SME extends the PPN formalism to allow an anisotropic breaking of the Lorentz symmetry in the preferred frame [7]. The action for the pure gravity sector within the minimal SME in Riemannian spacetime reads [6,7],

$$
S=\frac{1}{16 \pi G} \int\left[\sqrt{-g}\left(R-2 \Lambda-u R+s^{\mu v} R_{\mu v}^{\mathrm{T}}+t^{\kappa \lambda \mu \nu} C_{\kappa \lambda \mu \nu}\right)-V\left(g^{\mu \nu} ; u, s^{\mu v}, t^{\kappa \lambda \mu \nu}\right)\right] \mathrm{d}^{4} x,
$$

where the extra fields, $u, s^{\mu v}$, and $t^{\kappa \lambda \mu v}$ would have obtained their vacuum expectation values, $\bar{u}$, $\bar{s}^{\mu \nu}$, and $\bar{t}^{\kappa \lambda \mu v}$, respectively, through spontaneous symmetry breaking by minimizing the interaction potential term, $V\left(g^{\mu v} ; u, s^{\mu v}, t^{\kappa \lambda \mu v}\right)[6,7]$. At the first post-Newtonian approximation, only nine degrees of freedom in the dimensionless matrix, $\bar{s}^{\mu v}$, enter the actual dynamics of a gravitating system [7].

The two-body dynamics of a binary pulsar was worked out with osculating elements by Bailey and Kostelecký [7], and recently it was extended to include Lorentz-violating matter-gravity couplings [35] and higher-order curvature couplings [36]. Nevertheless, the timing formulae developed in $[7,35]$ contain strong degeneracy in parameters, and have not been used yet with real pulsar timing data. In general, LLIv terms introduce time variations in the orbital eccentricity, the longitude of periastron, and the orbital inclination for a binary pulsar. These contributions are controlled by the same set of underlying Lorentz-violating parameters from the SME effective action, but the magnitude of these contributions differs for different pulsars because of different projection effects (see [7]). They are caused by the different sky position, the different orbital orientation, and the different 3-dimensional binary velocities of pulsar systems. By combining different binary pulsar systems, it is possible to break the degeneracy and obtain stringent limits on all components of $\bar{s}^{\mu \nu}$ (except $\left.\bar{s}^{\mathrm{TT}}[18]\right)$.

Besides the modifications on the orbital dynamics of binary pulsars, SME coefficients, like in the PPN formalism, introduce a free precession to solitary pulsars $[7,18]$ that is inversely proportional to the spin period of pulsars. The precession is controlled by the same set of Lorentz-violating parameters, therefore, with careful studies, one can combine the tests from binary pulsars with the tests from solitary pulsars [18]. A first systematic study with different pulsars was carried out in [18] that combined thirteen best systems with twenty-seven different tests to break parameter degeneracy and reduce parameter correlations. Later, the Lorentzian boost effect from the movements of the pulsar systems with respect to the solar system was included to study the remaining unconstrained time-time component of $\bar{s}^{\mu \nu}$, namely $\bar{s}^{\mathrm{TT}}$ [19]. The limits on the SME coefficients obtained from pulsar systems turn out to be among the best limits for the pure gravity sector of the minimal SME [1,5]. In addition, these limits have promising prospects to be improved greatly in the future, scaling as $T_{\mathrm{obs}}^{3 / 2}$, where $T_{\mathrm{obs}}$ is the span of the observational time [18]. 


\section{Discussion}

LLI is an important concept of modern physics that deserves the most stringent examination from experiments. LLIv in the gravitational interaction, which is an important ingredient of the strong equivalence principle [2], is relatively harder to be studied than its flat-spacetime counterpart, because of the intrinsic weakness of gravitation (with an extremely small coupling constant, $G$ ). However, some trial theories, in seeking an ultimate quantum gravity, have motivated great interest in looking for possible signatures from LLIv [37]. Precision pulsar timing experiments turn out to be one of the best celestial laboratories to study the phenomena of LLIv in great detail, especially in the new era with giant radio telescopes, such as the Five-hundred-meter Aperture Spherical Telescope (FAST) and the Square Kilometre Array (SKA) [38]. If LLIv is discovered in the future, it will result in a paradigm shift in the fundamental physics; otherwise, the ever-tight new experimental limits of LLI are extremely useful to guide further development of alternative gravity theories.

Conflicts of Interest: The author declares no conflict of interest.

\section{References}

1. Kostelecký, V.A.; Russell, N. Data Tables for Lorentz and CPT Violation. Rev. Mod. Phys. 2011, 83, 11-31.

2. Will, C.M. The Confrontation between General Relativity and Experiment. Living Rev. Relativ. 2014, 17, 4.

3. Tasson, J.D. What Do We Know about Lorentz Invariance? Rep. Prog. Phys. 2014, 77, 062901.

4. Shao, L.; Wex, N. Tests of Gravitational Symmetries with Radio Pulsars. Sci. China Phys. Mech. Astron. 2016, 59, 699501.

5. Hees, A.; Bailey, Q.G.; Bourgoin, A.; Pihan-LeBars, H.; Guerlin, C.; le Poncin-Lafitte, C. Tests of Lorentz Symmetry in the Gravitational Sector. arXiv 2016, arXiv:1610.04682v1.

6. Kostelecký, V.A. Gravity, Lorentz Violation, and the Standard Model. Phys. Rev. D 2004, 69, 105009.

7. Bailey, Q.G.; Kostelecký, V.A. Signals for Lorentz Violation in Post-Newtonian Gravity. Phys. Rev. D 2006, $74,045001$.

8. Kostelecký, V.A.; Tasson, J.D. Matter-Gravity Couplings and Lorentz Violation. Phys. Rev. D 2011, 83, 016013.

9. Iorio, L. Constraining the Preferred-Frame $\alpha_{1}, \alpha_{2}$ Parameters from Solar System Planetary Precessions. Int. J. Mod. Phys. D 2014, 23, 1450006.

10. Iorio, L. Preliminary bounds of the gravitational local position invariance from Solar system planetary precessions. Mon. Notic. Roy. Astron. Soc. 2014, 437, 3482-3489.

11. Iorio, L. Orbital effects of Lorentz-violating standard model extension gravitomagnetism around a static body: A sensitivity analysis. Class. Quantum Gravity 2012, 29, 175007.

12. Battat, J.B.R.; Chandler, J.F.; Stubbs, C.W. Testing for Lorentz Violation: Constraints on Standard-Model-Extension Parameters via Lunar Laser Ranging. Phys. Rev. Lett. 2007, 99, 241103.

13. Bourgoin, A.; Hees, A.; Bouquillon, S.; Le Poncin-Lafitte, C.; Francou, G.; Angonin, M.-C. Testing Lorentz Symmetry with Lunar Laser Ranging. arXiv 2016, arXiv:1607.00294v3.

14. Müller, H.; Chiow, S.-W.; Herrmann, S.; Chu, S.; Chung, K.-Y. Atom-Interferometry Tests of the Isotropy of Post-Newtonian Gravity. Phys. Rev. Lett. 2008, 100, 031101.

15. Damour, T.; Esposito-Farèse, G. Testing Local Lorentz Invariance of Gravity with Binary-Pulsar Data. Phys. Rev. D 1992, 46, 4128-4132.

16. Shao, L.; Wex, N. New Tests of Local Lorentz Invariance of Gravity with Small-Eccentricity Binary Pulsars. Class. Quantum Gravity 2012, 29, 215018.

17. Shao, L.; Caballero, R.N.; Kramer, M.; Wex, N.; Champion, D.J.; Jessner, A. A New Limit on Local Lorentz Invariance Violation of Gravity from Solitary Pulsars. Class. Quantum Gravity 2013, 30, 165019.

18. Shao, L. Tests of Local Lorentz Invariance Violation of Gravity in the Standard Model Extension with Pulsars. Phys. Rev. Lett. 2014, 112, 111103.

19. Shao, L. New Pulsar Limit on Local Lorentz Invariance Violation of Gravity in the Standard-Model Extension. Phys. Rev. D 2014, 90, 122009.

20. Kostelecký, V.A.; Tasson, J.D. Constraints on Lorentz Violation from Gravitational Čerenkov Radiation. Phys. Lett. B 2015, 749, 551-559. 
21. Tasson, J.D. The Standard-Model Extension and Gravitational Tests. Symmetry 2016, 8, 111.

22. Le Poncin-Lafitte, C.; Hees, A.; Lambert, S. Lorentz symmetry and Very Long Baseline Interferometry. arXiv 2016, arXiv:1604.01663v1.

23. Bailey, Q.G.; Kostelecký, V.A.; Xu, R. Short-Range Gravity and Lorentz Violation. Phys. Rev. D 2015, $91,022006$.

24. Shao, C.-G.; Tan, Y.-J.; Tan, W.-H.; Yang, S.-Q.; Luo, J.; Tobar, M.E.; Bailey, Q.G.; Long, J.C.; Weisman, E.; $\mathrm{Xu}, \mathrm{R}$.; et al. Combined Search for Lorentz Violation in Short-Range Gravity. Phys. Rev. Lett. 2016, $117,071102$.

25. Long, J.C.; Kostelecký, V.A. Search for Lorentz violation in short-range gravity. Phys. Rev. D 2015, 91, 092003.

26. Shao, C.-G.; Tan, Y.-J.; Tan, W.-H.; Yang, S.-Q.; Luo, J.; Tobar, M.E. Search for Lorentz invariance violation through tests of the gravitational inverse square law at short ranges. Phys. Rev. D 2015, 91, 102007.

27. Wex, N. Small-Eccentricity Binary Pulsars and Relativistic Gravity. arXiv 2000, arXiv:gr-qc/0002032.

28. Xie, Y. Testing Lorentz Violation with Binary Pulsars: Constraints on Standard Model Extension. Res. Astron. Astrophys. 2013, 13, 1-4.

29. Will, C.M.; Nordtvedt, K., Jr. Conservation Laws and Preferred Frames in Relativistic Gravity. I. Preferred-Frame Theories and an Extended PPN Formalism. Astrophys. J. 1972, 177, 757-774.

30. Wex, N. Testing Relativistic Gravity with Radio Pulsars. arXiv 2014, arXiv:1402.5594v1.

31. Freire, P.C.C.; Wex, N.; Esposito-Farèse, G.; Verbiest, J.P.W.; Bailes, M.; Jacoby, B.A.; Kramer, M.; Stairs, I.H.; Antoniadis, J.; Janssen, G.H. The Relativistic Pulsar-White Dwarf Binary PSR J1738+0333-II. The Most Stringent Test of Scalar-Tensor Gravity. Mon. Notic. Roy. Astron. Soc. 2012, 423, 3328-3343.

32. Shao, L.; Wex, N.; Kramer, M. New Constraints on Preferred Frame Effects from Binary Pulsars. Proc. Int. Astron. Union 2012, 291, 496-498.

33. Nordtvedt, K. Probing Gravity to the Second Post-Newtonian Order and to One Part in $10^{7}$ Using the Spin Axis of the Sun. Astrophys. J. 1987, 320, 871-874.

34. Shao, L.; Wex, N. New Limits on the Violation of Local Position Invariance of Gravity. Class. Quantum Gravity 2013, 30, 165020.

35. Jennings, R.J.; Tasson, J.D.; Yang, S. Matter-Sector Lorentz Violation in Binary Pulsars. Phys. Rev. D 2015, 92, 125028.

36. Bailey, Q.G. Anisotropic Cubic Curvature Couplings. Phys. Rev. D 2016, 94, 065029.

37. Amelino-Camelia, G. Quantum-Spacetime Phenomenology. Living Rev. Relativ. 2013, 16, 5.

38. Shao, L.; Stairs, I.H.; Antoniadis, J.; Deller, A.T.; Freire, P.C.C.; Hessels, J.W.T.; Janssen, G.H.; Kramer, M.; Kunz, J.; Lämmerzahl, C.; et al. Testing Gravity with Pulsars in the SKA Era. arXiv 2015, arXiv:1501.00058v1. 